\title{
La inserción laboral de los titulados y tituladas de arte dramático de la ESADg
}

\author{
The employability of Dramatic Art Graduates in the ESAD of Galicia
}

\author{
Manuel F. Vieites*, María Dapía**, Reyes Fernández** \\ *ESAD de Galicia, **Universidade de Vigo
}

\begin{abstract}
Resumen
Diez años después de la creación de la Escuela Superior de Arte Dramático de Galicia, y pasados cinco desdeque la Ley Orgánica de Educación integrase en 2010 estas enseñanzas en el Espacio Europeo de Educación Superior, se analiza el proceso de inserción laboral de los titulados superiores que cursaron sus estudios al amparo de la LOGSE, entre 2005 y 2013. Para ello se ha diseñado un cuestionario específico orientado a analizar cuestiones relativas a la formación recibida, a los procesos de tránsito a la vida activa, y a los ámbitos de actividad profesional más importantes, que ofrece resultados de especial relevancia para el futuro de la institución.

Palabras clave: inserción laboral, arte dramático, formación.
\end{abstract}

\begin{abstract}
Ten years after the creation of the Galician School of Dramatic Art, and being five years since the Educational Act of 2006 integrated in 2010 those courses into the European Higher Education Area, this paper analyses the processes of employment of the LOGSE graduates that followed their courses between 2005 and 2013. Therefore, we have designed a specific questionnaire aimed at analyzing issues related to the training received, the processes of transition to working life, and the principal areas of professional activity, and the results obtained provide information of particular relevance for the future of the institution.
\end{abstract}

Keywords: Employment, Theatre Arts, Education.

\section{Introducción}

La Ley Orgánica de Ordenación General del Sistema Educativo (LOGSE) supuso un punto de inflexión muy positivo para las enseñanzas artísticas superiores que, pese a lo dispuesto en la Ley General de Educación de 1970, se habían quedado fuera de la universidad. El Real Decreto 754/1992 establecía los contenidos mínimos del plan de estudios de un título superior que se equiparaba a todos los efectos al de una licenciatura universitaria. Ese sería el marco legislativo bajo el que en 2005 la Consejería de Educación y Ordenación Universitaria publicaba el Decreto 220/2005 que regulaba el currículo de los estudios superiores de arte dramático que en ese mismo año se comienzan a impartir en la Escuela Superior de Arte Dramático de Galicia, creada por la citada Consejería mediante el Decreto 145, de 2 de junio de 2005.

En septiembre de 2005 comenzaban su periplo formativo las primeras promociones en las especialidades de dirección escénica e interpretación textual, a las que se sumarían más tarde las especialidades de dramaturgia y escenografía. La ESAD de Galicia configuraba así en pocos años una oferta educativa llamada a satisfacer las demandas de formación de un ámbito de la creación artística especialmente relevante en Galicia desde los años ochenta y noventa del pasado siglo XX (Fernández Vieites, 2012).

Desde entonces, y hasta el curso escolar 2012-2013, terminan sus estudios cinco promociones, lo que implica un total de 135 personas. Actualmente, una vez puesto en marcha el nuevo plan de estudios regulado por el RD $1614 / 2009$ y el RD 630/2010, parece oportuno analizar aspectos básicos de la formación y la transición de esas cinco primeras promociones tanto por los datos que puedan aportar en relación a su incorporación a la práctica profesional y a cuáles sean los ámbitos más relevantes en la misma, como por la valoración que pueden hacer de su formación y de la utilidad que pueda haber tenido en esa transición a la vida activa.

El hecho de que los estudios superiores de arte dramático se hayan mantenido fuera de la universidad, y por tanto alejados de prácticas propias de esta institución como la promoción del empleo de sus titulados y tituladas, es una de las razones de que en los centros que imparten estos estudios apenas exista una cultura orientada al desarrollo de estudios sobre la empleabilidad, si bien exista alguna excepción interesante (Domínguez Amorós y Coco, 1999).

No quiere ello decir que no se hayan publicado algunos trabajos sobre la cuestión,si bien tengan carácter genérico. Así, en el número correspondiente al primer trimestre de 2010, el boletín informativo Darse, que publica la RESAD de Madrid, se abría con un breve editorial en el que Ángel Martínez Roger, director del centro, formulaba toda una serie de retos relativos al futuro profesional de los titulados en arte dramático y lo hacía en los siguientes términos: "Además de los teatros privados y de las sedes escénicas de las tres administraciones, cumpliendo las funciones obvias que nos son propias, está la aplicación de nuestras disciplinas a los muy diferentes ámbitos; la cultura: museos, turismo en patrimonio, ocio; lo sanitario..., acción en hospitales...; lo social: teatro foro, teatro encuentro, centros de día; la juventud..., monitores, guías de animación y tiempo libre; la inmigración: acciones para integración; la didáctica...; las instituciones penitenciarias...; la ayuda a la formación del empresariado" (p. 3).

$\mathrm{Su}$ propuesta estaba en plena consonancia con algunas experiencias de apertura de nuevos ámbitos de ejercicio profesional que se desarrollan en todo el Estado y que 
apuntan en la dirección de ampliar un campo profesional limitado incomprensiblemente a lo escénico y que presenta posibilidades ciertas atendiendo a la formación inicial que reciben los estudiantes de arte dramático y que a buen seguro se podría y debiera complementar de formas diversas, y no sólo a través de los estudios de postgrado. Pero se trata de posibilidades para aumentar también la visibilidad del teatro y su legitimidad.

En esa misma dirección, en el año 2008la Agencia Galega das Industrias Culturais, publicaba el informe Das funciónssociais e dos novosespazosprofesionais para as artes escénicas en Galicia, en el que en un plano más bien teórico aunque asentado en un conocimiento sólido del campo, se señalaban algunas áreas de especial relevanciapara el ejercicio profesional:

- El ámbito de la creación teatral de carácter general.

- El ámbito de la creación teatral en ámbitos deficitarios como puede ser el del teatro para la infancia y la juventud.

- El ámbito de la gestión artística y cultural.

- El ámbito de la educación y de la formación teatral formal y no formal.

- El ámbito de los servicios culturales, de la acción cultural y de la animación sociocultural.

- El ámbito de las ciencias de la salud, de la prevención, de la terapia..., en el que los titulados en arte dramático se integran en un equipo de trabajo bajo la coordinación y la supervisión de un terapeuta.

- El ámbito de la investigación, relacionada con lo que se ha denominado $\mathrm{I}+\mathrm{i}+\mathrm{C}$, una investigación para la innovación y para la creación (Pose et al., 2008, 47 e ss.).

Si bien la peripecia conocida de numerosas personas tituladas en arte dramático mostraba la diversidad de oportunidades laborales establecida en ese estudio, faltaban datos empíricos que certificasen el alcance real de la misma, por lo que se hacía necesario realizar un estudio empírico sistemático.

Por otro lado, en noviembre de 2014 la Consellería de Cultura, Educación y Ordenación Universitaria y la Axencia para a Calidade do Sistema Universitario de Galicia firmaban un convenio de colaboración para que la ACSUG realizase la acreditación de las titulaciones artísticas superiores, y entre ellas los estudios superiores de arte dramático que ofrece la ESAD de Galicia, equivalentes a todos los efectos a las titulaciones oficiales de grado.

En ese doble contexto, marcado por la necesidad de conocer ámbitos de empleo de laspersonas tituladas y al mismo tiempo cumplir los requisitos de la acreditación,parecía obligado que la ESADg analizase los resultados de su acción educativa, valorando no solo la idoneidad del plan de estudios en función de las demandas del ejercicio profesional, sino también en función de yacimientos de empleo no siempre adecuadamente visualizados, pero no por ello inexistentes. Ese es el marco en el que hay que situar el presente estudio, del que ahora ofrecemos algunos resultados de carácter genérico.

Por ello en esta primera comunicación pública de resultados nos hemos propuesto mostrarlas características básicas del proceso de transición a la vida activadel alumnado (grado de inserción, condiciones laborales...), y conocer la valoración que el mismo hace de la formación recibida en la ESADg así como de las necesidades detectadas en ese tránsito.

\section{Método}

\section{Muestra}

Para la realización de este estudio hemos invitado a participar a todos los egresados y egresadas desde la primera promoción, según el plan de estudios LOGSE de 2005, en vigor hasta el año 2103, que cursan 5 promociones que constituyen un total de 135 personas. Finalmente, se han obtenido un total de 42 respuestas, lo que supone más del $30 \%$ de la población objeto de estudio.

El perfil de la muestra está definida por ser mayoritariamente mujer $(71.4 \%)$, con una media de edad de $29.16\left(\mathrm{~S}_{\mathrm{x}}=6,80\right)$ y un rango entre 22 y 59, habiendo realizado las siguientes especialidades: dirección escénica y dramaturgia $21.5 \%$, interpretación $69 \%$ y escenografía $9.5 \%$.

\section{Instrumento}

Para llevar a cabo la recogida de datos se elaboró un cuestionario diseñado ad hoc. En su diseño, se han incluido aspectos de interés según los objetivos del estudio. El instrumento agrupa los ítems, fundamentalmente de tipo cerrado, en tres partes: un apartado de datos sociodemográficos y académicos, una segunda sobre aspectos referidos a la formación recibida $\mathrm{y}$, en último lugar, un bloque relativo a las características del empleo actual.

La primera parte incluía cuestiones sobre edad, género, procedencia, especialidad cursada, año de graduación...; la segunda estaba integrada por un conjunto de cuestiones diseñadas para recoger información relativa a la formación recibida: satisfacción, propuestas de mejora, valoración,... y en tercer lugar, la última incluía todo aquello que tiene que ver con su empleo actual: inserción, vinculación con la formación, ingresos, tipología de contrato, ámbito de actuación,...

\section{Procedimiento}

A través de la dirección de la ESADg, y con la colaboración de la Secretaría del centro, nos pusimos en contacto vía email con todos los egresados para comunicarles los objetivos del estudio y los instrumentos a aplicar. Se les solicitó su participación anónima y voluntaria, así como sinceridad en sus respuestas. Aquellos que aceptaron participar podían hacer su entrega vía email o correo postal. 


\section{Diseño}

Se trata de un estudio de corte transversal, con una metodología que se caracteriza por "describir poblaciones sin que en su planteamiento se incluyan hipótesis propiamente dichas" (Montero y León, 2005, p. 119). De este modo, en este tipo de estudios se miden de forma independiente sus variables, para luego integrar dichas mediciones y decir cómo es y se manifiesta el fenómeno de interés.

\section{Análisis de datos}

Para el análisis de datos se recurrió principalmente a la utilización de técnicas de estadística descriptiva univariada, utilizando estadísticos de tendencia central y de dispersión (medias y desviaciones típicas), análisis de frecuencias y porcentajes. Debido al número de respuestas obtenida no se consideró oportuno llevar a cabo contrastes analíticos. Los datos fueron analizados mediante el programa estadístico SPSS 20.0 para Windows.

\section{Resultados y discusión}

De los resultados obtenidos extraemos importantes conclusiones relativas a la formación recibida y al ejercicio profesional.

Tabla 1.

Valoración formación ESADg

\begin{tabular}{llc}
\hline $\begin{array}{l}\text { Aspectosvalorad } \\
\text { os }\end{array}$ & \multicolumn{1}{c}{$\begin{array}{c}\text { Opciones } \\
\text { respuesta }\end{array}$} & $\%$ \\
\hline Satisfacción & Baja & 9.8 \\
formación & Regular & 48.8 \\
& Alta & 39.0 \\
& Muy Alta & 2.4 \\
\hline Valoración & Insuficiente & 26.3 \\
formación & Suficiente & 42.1 \\
& Buena & 26.3 \\
& Muy Buena & 5.3 \\
\hline Carácter & Practica & 7.1 \\
formación & Teórico-practica & 64.3 \\
& Teórica & 11.9 \\
& Muy teórica & 16.7 \\
\hline Inclusión & Prácticas & 81.1 \\
materias & Teóricas & 5.4 \\
& Ninguna & 13.5 \\
\hline Repetir matrícula & Si & 70.0 \\
& No & 30.0 \\
\hline Recomendación & Si & 83.7 \\
& No & 16.3 \\
\hline
\end{tabular}

En relación a la formación, como se puede ver en la Tabla 1, se percibe una satisfacción medio-alta, siendo únicamente valorada de forma negativa por un $9.8 \%$; así mismo, sólo un $23.8 \%$ considera que la formación recibida en la ESADg atendiendo al desempeño profesional fue insuficiente y para más del $30 \%$ ha sido buena o muy buena. Para un $64.3 \%$, la formación recibida en el ESADg tuvo una orientación teóricopráctica, aunque para un porcentaje nada despreciable, más del $25 \%$, recibe la consideración de teórica o muy teórica. En coherencia con esta valoración un $81.1 \%$ plantea como mejora en su formación la inclusión de más materias prácticas.

Esta valoración positiva de la formación en la ESADg se ve reflejada en las cuestiones planteadas acerca de si volverían a matricularse y si recomendarían el centro a quien quisiera estudiar arte dramático; el 70\% repetiría la matrícula y el $83.7 \%$ lo recomendarían.

Asimismo, se ha preguntado al alumnado egresado acerca de qué factores podrían explicar el grado de satisfacción con la formación recibida en la ESADg. Como puede apreciarse en la Figura 1, los tres elementos analizados -profesorado, plan de estudios, recursos e instalaciones- reciben una valoración positiva. Así, la mayor fuente de satisfacción reside en el profesorado (más del $60 \%$ lo considera un factor bastante o muy influyente en su satisfacción) y, por el contrario, la menor le corresponde a la opción recursos e instalaciones. El plan de estudios aglutina un mayor número de respuestas en la categoría regular.

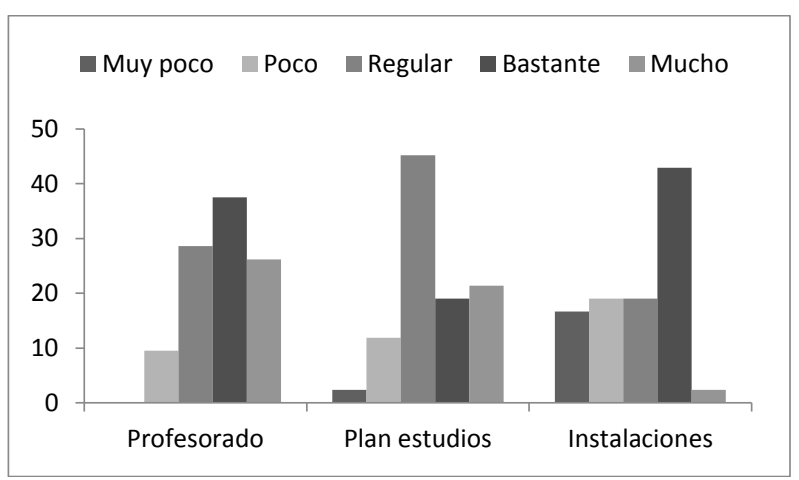

Figura 1. Factores influyentes satisfacción formación ESADg

Los datos referidos al ejercicio profesional se encuentran recogidos en la Tabla 2, y se han subdividido en aquellos centrados en describir la situación laboral de los egresados y egresadas de la ESADg, los destinados a configurar las condiciones laborales en las que desempeñan su empleo y los que trazan los rasgos básicos de las entidades contratantes.

Este fue uno de los ámbitos en los que la dirección de la ESAD de Galicia mostraba un mayor interés, en tanto las áreas preferentes en las que ejercen su actividad profesional los titulados y tituladas deben tener una correspondencia curricular para el desarrollo de las competencias buscadas por el mercado laboral, especialmente en un momento en que la superación de la acreditación podría acelerar la adscripción y posterior integración de los estudios superiores de arte dramático en la universidad, lo que implicaría desarrollar un nuevo plan de estudios, al que incorporar mejoras derivadas de las conclusiones de estudios como el presente.

En sintonía con trabajos ya señalados (Domínguez Amorós y Coco, 1999; Pose y cols., 2008), se trataba de conocer si las percepciones derivadas de la peripecia profesional observada en no pocos titulados en arte dramático que habían estudiado en las escuelas superiores de Madrid, Barcelona, Murcia o Málaga, se podía confirmar con datos contrastados. Por otra parte, también se hacía necesario conocer el impacto de cada 
sector, especialmente el de dos especialmente relevantes como son la producción audiovisual (fundamentalmente cine y series de televisión) o la educación teatral no formal o la animación sociocultural o teatral.

Tabla 2.

Ejercicio profesional ESADg

\begin{tabular}{|c|c|c|c|}
\hline \multicolumn{2}{|c|}{ Aspectos valorados } & \multirow{2}{*}{$\begin{array}{l}\text { Opciones } \\
\text { respuesta }\end{array}$} & \multirow[t]{2}{*}{$\%$} \\
\hline Situación & Relación & & \\
\hline \multirow[t]{8}{*}{ laboral } & laboral & Cont. Temporal & 40 \\
\hline & & Sin contrato & 5 \\
\hline & & Autoempleo & 30 \\
\hline & & Buscando & 20 \\
\hline & Vínculo & $\mathrm{Si}$ & 60.6 \\
\hline & formación & No & 39.4 \\
\hline & Duración & Días & 304 \\
\hline & Ingresos & & $1.058 €$ \\
\hline \multirow{18}{*}{$\begin{array}{l}\text { Condicio- } \\
\text { nes } \\
\text { laborales }\end{array}$} & Funciones/ & $\mathrm{Si}$ & 89.5 \\
\hline & categoría & No & 10.5 \\
\hline & Titulación & $\mathrm{Si}$ & 50 \\
\hline & ESADg & No & 50 \\
\hline & Jornada & Completa & 37.4 \\
\hline & & Media & 31.3 \\
\hline & & Días/temporada & 31.3 \\
\hline & Ámbitos & Interpretación & 54.8 \\
\hline & profesio- & Dir. Escena & 21.4 \\
\hline & nales & Dramaturgia & 16.7 \\
\hline & & Diseño escénico & 7.1 \\
\hline & & Producción & 21.4 \\
\hline & & Gestión & 16.7 \\
\hline & & Formación & 28.6 \\
\hline & & Animación & 26.2 \\
\hline & & Audiovisual & 38.1 \\
\hline & & Téc. escena & 12 \\
\hline & & Otros & 16.7 \\
\hline \multirow{7}{*}{$\begin{array}{l}\text { Entidad } \\
\text { emplea- } \\
\text { dora }\end{array}$} & Carácter & Público & 33.4 \\
\hline & & Privado sí subv. & 33.3 \\
\hline & & Privado no subv & 33.3 \\
\hline & CNAE & Artes escénicas & 62,5 \\
\hline & & Educación Sec. & 12,5 \\
\hline & & Otra educación & 12,5 \\
\hline & & Otros & 12,5 \\
\hline
\end{tabular}

La situación laboral de los egresados y egresadas de la ESADg se caracteriza por la presencia de un porcentaje alto en su nivel de contratación $(80 \%)$, una adecuación elevada entre el trabajo desempeñado y la formación recibida $(60.6 \%)$, una duración media de 304 días y unos ingresos mensuales medios de $1.057 €$.

Las condiciones en las que estaban desempeñando su labor profesional son las siguientes: categoría profesional acorde con las funciones desempeñadas $(89.5 \%)$, exigencia de la titulación obtenida en la ESADg sólo en el $50 \%$ de los casos, alta variabilidad respecto a las jornadas laborales realizadas y gran diversidad de los ámbitos profesionales en los que desarrollan su labor, preferentemente interpretación (54.76\%), audiovisual (38.09\%), formación (28.57\%), animación $(26.19 \%)$ y con menor presencia producción, dirección de escena, gestión, dramaturgia y diseño escénico.
Por último, las entidades que han empleado a los egresados y egresadas de la ESADg se caracterizan por ser mayoritariamente de titularidad privada $(66.6 \%)$, si bien la mitad recibieron una subvención pública para poder desarrollar la actividad para la que estaba contratada la persona encuestada, y de desarrollar su actividad económica, según la clasificación de la CNAE, en el ámbito de las artes escénicas (62.5\%), seguida por el ámbito educativo (25\%).

\section{Conclusiones}

Como conclusiones más significativas de este estudio quisiéramos desatacar la importancia y necesidad de realizar este tipo de estudios que ayudan a perfilar las profesiones, así como plantear propuestas de mejora. Esto es especialmente relevante en el caso de profesiones que aglutinan una multiplicidad de ocupaciones y ámbitos de actuación que todavía se están definiendo y conquistando. Ésta es una de las mayores dificultades del trabajo realizado; nos hemos centrado en los titulados y tituladas por la ESADg, aunque la diversidad de ocupaciones que incluye el sector de las artes escénicas está siendo desempeñadas también por otras personas, tituladas o no tituladas.

A pesar de las limitaciones, en este estudio se indican tendencias que exigen nuevas investigaciones y análisis; se podrían ampliar los informantes, en número y tipología, las técnicas de recogida de datos, combinando análisis cualitativos y cuantitativos,...

La formación de la ESADg recibe una valoración positiva y el grado de satisfacción de sus egresados es elevado; plantean como propuesta de mejora incrementar el número de materias de tipo práctico, y ahondar en la dimensión práctica de las existentes. La mejora de la formación es uno de los retos de todo proceso formativo y, por tanto, este estudio aporta datos de interés para el análisis de su plan de estudios. Todo ello tiene todavía mayor interés en un momento en el que la titulación busca mayor dignificación, apostando por su entrada en la universidad.

Debido a que, a la mitad de los egresados y egresadas de la ESADg, se les ha exigido en su proceso de incorporación al mercado laboral el título obtenido en este centro, a que las funciones desarrolladas son acordes con la categoría profesional desempeñada y a que las condiciones en que lo han hecho implica, en la gran mayoría de los casos, la existencia de un vínculo contractual legal, podemos calificar su inserción como buena, si bien peca de excesiva temporalidad en los contratos formalizados, tanto en lo referido a su duración como a la jornada.

Así mismo, podemos corroborar las conclusiones de los diversos estudios aquí citados referidas a que el campo profesional de estos titulados y tituladas se está ampliando, y ya no está limitado a lo escénico. En esa dirección, los datos del estudio permiten configurar cuatro grandes áreas, como son la creación escénica en diversos campos de especialización, la formación y la animación, la creación audiovisual, y la gestión.

Por último, y a pesar del descenso de las subvenciones públicas en general durante los últimos cuatro años, y en 
particular de las relativas a la creación y la difusión cultural, la contratación de estos y estas profesionales sigue dependiendo de lo público, aun cuando la titularidad de las entidades contratantes sea mayoritariamente de carácter privado.

\section{Referencias}

Domínguez Amorós, M. y Coco, A. (1999). Insercions i trajectòriessocioprofessionals en el sector de les artsescèniques. Revista catalana de sociología, 10, 151-186.

Fernández Vieites, M. (2010). As artes escénicas en Galicia. Elementos para unhadiganose. En Víctor F. Freixanes y Alberto Meixide (eds.), O Capital da cultura: unhaachegaás industrias culturais de Galicia (pp. 463-537). A Coruña: Fundación Caixa Galicia.
Martínez Roger, A. (2010). Editorial. Darse, 5, p. 3.http://www.resad.es/noticias/darse5.pdf

Montero, I., y León, O. (2005). Sistema de clasificación del método en los informes de investigación en psicología. Revista Internacional de Psicología Clínica y de la Salud/ International.Journal of Clinical and HealthPsychology, 5, 115-127.

Pose, H.M., Caride, J.A., Gradaille, R. yVieites, M.F. (2008). Das funciónssociais e dos novosespazosprofesionais para as artes escénicas en Galicia. -Santiago: AGADIC.

http://www.agadic.info/temas/agadic/gfx/publicacion/ Das_funcions_sociais.pdf 